\title{
Combination of Adaptive Resonance Theory 2 and RFM Model for Customer Segmentation In Retail Company
}

\author{
I Ketut Gede Darma Putra \\ Department of Information \\ Technology \\ Udayana University, Bali, \\ Indonesia
}

\author{
A. A. Kt. Agung Cahyawan \\ Department of Information \\ Technology \\ Udayana University, Bali, Indonesia
}

\author{
Dian Shavitri $\mathrm{H}$. \\ Department of Information \\ Technology \\ Udayana University, Bali, \\ Indonesia
}

\begin{abstract}
Customer segmentation is one of the important issues in Customer Relationship Management (CRM). This paper demonstrated the fusion of ART 2 algorithm and RFM (Recency, Frequency, and Monetary) model to cluster the customers in Retail Company. Each cluster is validated by searching the overall average value of silhouette index. In this paper used 17.999 rows of data transaction then was modeled into RFM model and become 499 rows of RFM data. Experiments were done by forming 2 to 6 clusters by changing the value of vigilance parameter $(\rho)$ and noise suppression $(\theta)$.

In this paper, all clusters formed have overall average Silhouette index value more than 0 , especially for $2,3,4$ clusters formed have overall average Silhouette index value close to 1 , indicates most of the silhouette value in all clusters were formed have a positive value or above zero, its means ART 2 clustering algorithm which produces two to six clusters have been able clustering well.
\end{abstract}

\section{General Terms}

CRM, Data Mining, Clustering

\section{Keywords}

Customer Segmentation, RFM Model, ART 2 Algorithm, Silhouette Index

\section{INTRODUCTION}

In the middle of a tight business competition nowadays, retail companies have shifted their attention from simply developing products and superior service to create personalized experiences of customer. This can be done with a full awareness that the relationship between companies and customer is essential to support the development and sustainability of the company. According to the Pareto law (the law 80:20), only $20 \%$ of all customers who represent $80 \%$ of corporate revenue. In this case, different customers represent different values. The management of retail companies should be able to recognize the best customer and trust the customer by increasing the company understanding of customers needs as individual so loyalty of the company can be maintained.

CRM (Customer Relationship Management) is the strategy for building, managing, and strengthening loyal and long-lasting customer relationships. CRM should be a customer-centric approach based on customer insight. Its scope should be the

\begin{abstract}
"personalized" handling of customers as distinct entities through the identification and understanding of their differentiated needs, preferences, and behaviors [1]. CRM is seen not only as a product or a service, but also a business philosophy that aims to maximize long-term customer value (customer lifetime value). By applying data mining technology and the concept of CRM, company can identify customers by segmenting customers. The purpose of customer segmentation process is to provide an assessment of the customers (customer scoring) and determines profile of the customer [2], so a company can determine customer behavior and implement appropriate marketing strategies to maximize profit for the company.
\end{abstract}

Analysis of data mining in relation to explore the customer relationship has been done in many researches. Various algorithms and methods are used e.g. the research to segment bank customer based on data mining [3], applying data mining with A Novel Utility and Frequency Based Item set Mining for improving CRM in retail business [4]. Many researches related to the RFM model also has been done a lot e.g. by combining RFM theory with K-means and Rough Set Theory to segment customers in an electronics company [5], combining RFM model with FCM and Fuzzy Subtractive algorithm in retail company [6] and also combining the use of RFM and bandwidth usage with K-Means to determine the customer segmentation in a telecommunication company [7], for ART 2 algorithm has been applied to determine the strategy for acquiring customer requirement patterns using ART 2 neural network is coupled with laddering technique [8].

There is no data mining tools for managing customer relationship works perfectly, therefore in this paper is worth to explore combination of ART 2 algorithm with RFM model then uses Silhouette algorithm to evaluate the clusters.

\section{METHODOLOGY}

The overview diagram of this research is shown in Figure 1. 


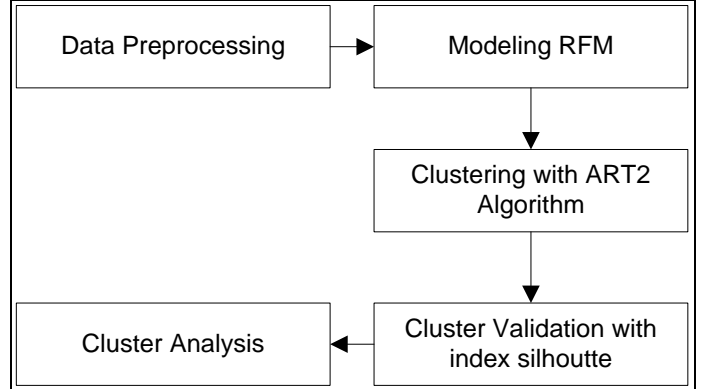

Figure 1. Research overview diagram

\subsection{Data Preprocessing}

In this paper uses data from a retail company $X$ which specialize to sell ingredients and baking equipment, from 2006 until 2010 retail company X has 1100 customers, 10.000 items and up to 100.000 number of transaction, to demonstrate the performance of RFM model and ART 2 algorithm uses data in 01/01/2008 to 31/12/2008 which consist of 17.999 rows of data transaction then was modeled into RFM model and become 499 rows of RFM data.

Processes in this step include selection of data used and ensure the quality of data. At this stage the problem to be faced is the noisy data and missing values. Data preprocessing process makes clustering process can be done easily and effectively. Table 1 shows the pieces of data used before RFM transformation.

Table 1. The pieces of data used before RFM transformation

\begin{tabular}{|c|c|c|}
\hline $\begin{array}{c}\text { Customer } \\
\text { Id }\end{array}$ & $\begin{array}{c}\text { Transaction } \\
\text { Date }\end{array}$ & $\begin{array}{c}\text { Transaction } \\
\text { Amount }\end{array}$ \\
\hline$\ldots \ldots$ & $\ldots \ldots$ & $\ldots \ldots$ \\
$\mathrm{C} 0219$ & $2008-11-17$ & 155500000 \\
$\mathrm{C} 0308$ & $2008-02-28$ & 150570000 \\
$\mathrm{C} 0359$ & $2008-01-30$ & 123175000 \\
$\mathrm{CO} 311$ & $2008-02-25$ & 100980000 \\
$\mathrm{C} 0203$ & $2008-02-29$ & 86650000 \\
$\mathrm{C} 0203$ & $2008-01-30$ & 86650000 \\
$\mathrm{C} 0109$ & $2006-04-29$ & 2752250 \\
$\mathrm{C} 0246$ & $2006-05-06$ & 2869000 \\
$\mathrm{C} 0117$ & $2006-05-06$ & 16486240 \\
$\ldots \ldots$. & $\ldots \ldots$. & $\ldots \ldots$. \\
\hline
\end{tabular}

Tabel 2. The pieces of RFM data used

\begin{tabular}{|c|c|c|c|c|}
\hline Customer Id & Recency & Frequency & Monetary & Year \\
\hline$\ldots \ldots$ & $\ldots \ldots$ & $\ldots \ldots$ & $\ldots \ldots$ & $\ldots \ldots$ \\
$\mathrm{C} 0234$ & 364 & 118 & 240179800 & 2008 \\
$\mathrm{C} 0337$ & 13 & 2 & 8357500 & 2008 \\
$\mathrm{C} 0752$ & 347 & 120 & 2886000 & 2008 \\
$\mathrm{C} 0221$ & 347 & 153 & 6136500 & 2008 \\
$\mathrm{C} 0026$ & 365 & 175 & 256950800 & 2008 \\
$\mathrm{C} 0081$ & 353 & 111 & 4134900 & 2008 \\
$\mathrm{C} 0050$ & 365 & 236 & 176860250 & 2008 \\
$\ldots \ldots$ & $\ldots \ldots$ & $\ldots \ldots$ & $\ldots \ldots$ & $\ldots \ldots$ \\
\hline
\end{tabular}

\subsection{Modeling RFM}

RFM model divides a large data into three aspect dimensional variables namely, Recency(R), Frequency $(F)$ and Monetary (M) [9]. The complete description is described below:

1. Recency - Recency refers to the time interval between the last transactions was conducted by customer until the present time or within a certain period. The closer interval time between last times purchasing with the present produce the higher value of Recency(R).

2. Frequency - Frequency refers to total number of transaction within a certain period. The larger number of transaction (frequently transaction) makes the higher value of Frequency $(\mathrm{F})$.

3. Monetary - Monetary refers to the amount of money was spent on a transaction within a specific time period. The larger amount of money was spent makes the higher value of Monetary (M).

There are six segments of customer for retailer through clustering [1], they are:

1. Superstar Customers

Customers in this class are the most loyal customers with highest value, with increasing number of visits and high transaction spending.

2. Golden Customers

Golden customers are the second highest value customers with increase number of visits and average transaction spending.

3. Typical Customers

Typical customers are customers who have average value with average frequency and average transaction spending.

4. Exceptional Occasions Customers

Exceptional Occasions customers are customers whose have low frequency, the second lowest after dormant customers, large basket and have a long time transaction after they last visit.

5. Everyday Shoppers

Everyday Shoppers are customers with increasing number of visits but low in transaction value.

6. Dormant Customers

Dormant customers are the lowest class, customers in this class have the lowest values, transaction amount and frequency did transaction long time after they last visit.

The pieces of data used in Table 1 will be modeled into RFM model (see Table 2). Value of Recency is taken by searching the time interval between the last transaction date until the end of period is set, Frequency is taken by summing the number of times the transaction is done by a customer until the end of period is set and for Monetary is taken by summing the customers transaction amount until the end of period is set. A certain weight for $\mathrm{R}, \mathrm{F}$, and $\mathrm{M}$ can be set, but in this paper uses a same weight to perform the clustering process.

\subsection{Clustering with ART 2 Algorithm}

Adaptive resonance architecture is unsupervised learning neural network that self-organized stable recognition codes in real time in response to arbitrary sequence of input pattern, is shown in figure 2 [10]. ART2 is a part of the ART algorithm used to perform continuous pattern input [11] which can automatically classify arbitrary input pattern as arbitrary noise input while suppressing an arbitrary [12]. 


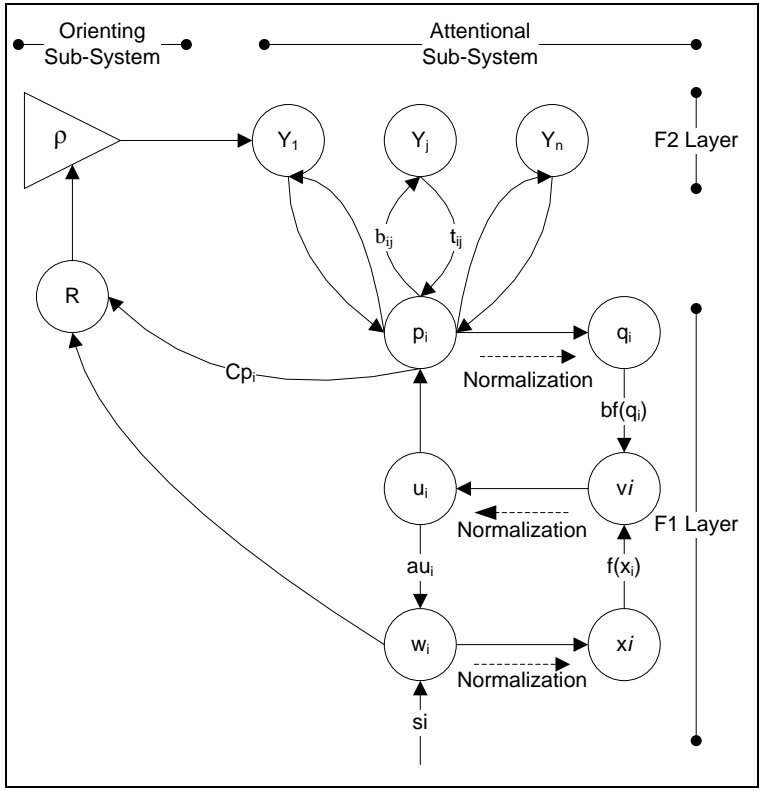

Figure 2. Typical Architecture of ART 2 [10]

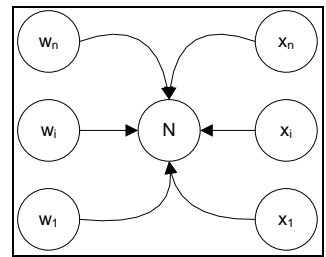

Figure 3. Additional detail between unit type $w$ and $x$

ART 2 architecture is basically formed by three groups of neurons, the input layer (F1 Layer), cluster layer (F2 Layer), and the layer to control the degree of patterns similarity placed on the same cluster (Reset Layer). Each unit in F1 layer is connected to each unit in F2 layer by using two types of weights, bottom-up weights $\left(b_{i j}\right)$ to connect the F2 layer with F1 Layer and top-down weights $\left(t_{j i}\right)$ to connect the F2 layer with the F1 layer.

Layer F1 on ART 2 consists of six types of unit, $w, x, u, v, p$ and $q$. Computation starts from $u$ unit then the signals from each $u$ unit are sent to $w$ unit and $p$ unit. $w$ unit sums the signals from $u$ unit and input signal $s$ then $p$ unit sums signals from $u$ unit and top-down weight if there is an active F2 unit. Activation $x$ and $q$ unit are the normalization of $w$ and $p$ unit (detail relationships of normalization from $w$ to $x$ unit is illustrated in Figure 3 [12] then an activation function are applied before the signal is sent to $v$ unit. $v$ unit will sum the signals which are received from $x$ and $q$ unit. F1 layer activation function only takes action to any signals which is less than noise suppression value $(\theta)$, after $F 1$ unit reach equilibrium then $p$ unit sends signals to $\mathrm{F} 2$ layer. Competition between cluster occurs in F2 layer, cluster with the largest network input unit will be the candidate to learn the input pattern, a cluster unit has an opportunity to learn the input patterns only if the input pattern can through the vigilance parameter $(\rho)$ is set, this decision was made at reset layer by accepting signals from F1 Layer. If a cluster unit is not permitted to learn the input pattern then another cluster unit will be selected as the next candidate. The algorithm of ART $2[10][11]$ is described below :

0 . Initialize parameter $a, b, \theta, c, d, e, \propto \rho$

1. Repeat step 2-9 until $n$-epoch
2. Update F1 layer

$$
\begin{aligned}
u_{i} & =\frac{v_{i}}{e+\|v\|} \\
w_{i} & =s_{i}+a u_{i} \\
p_{i} & =u_{i} \\
x_{i} & =\frac{s_{i}}{e+\|s\|} \\
q_{i} & =\frac{p_{i}}{e+\|p\|} \\
V_{i} & =f\left(x_{i}\right)+b f\left(q_{i}\right)
\end{aligned}
$$

3. Compute signals to F2 Layer

$$
y_{j}=\sum_{i} b_{i j} p_{i}
$$

4. Find the largest unit $y_{j}$ in F2 layer

5. Test for reset

$$
\begin{aligned}
& u_{i}=\frac{v_{i}}{e+\|v\|} \\
& p_{i}=u_{i}+d t_{j i} \\
& r_{i}=\frac{u_{i}+c p_{i}}{e+\|u\|+c\|p\|}
\end{aligned}
$$

$$
\text { if } \begin{aligned}
\|\mathrm{r}\| & <y_{j}=-1 \text { repeat step 5; if }\|\mathrm{r}\|>\rho, \\
W_{i} & =s_{i}+a u_{i} \\
x_{i} & =\frac{s_{i}}{e+|| s||} \\
q_{i} & =\frac{p_{i}}{e+|| p \| \mid} \\
V_{i} & =f\left(x_{i}\right)+b f\left(q_{i}\right)
\end{aligned}
$$

6. Repeat step 8,9 until weight equilibrium

7. Update bottom-up and top-down weight

$$
\begin{aligned}
& t_{j i}=\propto d u_{i}+\{1+\propto d(d-1)\} t_{j i} \\
& b_{j i}=\propto d u_{i}+\{1+\propto d(d-1)\} b_{j i}
\end{aligned}
$$

8. Update F1 layer units

$$
\begin{aligned}
u_{i} & =\frac{v_{i}}{e+\|v\|} \\
w_{i} & =s_{i}+a u_{i} \\
p_{i} & =u_{i}+d t_{j} \\
x_{i} & =\frac{s_{i}}{e+\|s\|} \\
q_{i} & =\frac{p_{i}}{e+\|p\|} \\
V_{i} & =f\left(x_{i}\right)+b f\left(q_{i}\right)
\end{aligned}
$$

Based on R, F, M data are shown in Table 2 clustering process with ART 2 algorithm will be conducted. Clustering will be done by dividing data into 2 to 6 clusters. Experiments are conducted by changing the value of vigilance parameter $(\rho)$ and noise suppression $(\theta)$ and see the results are obtained.

\subsection{Cluster Validation}

After clustering process to conduct 2 to 6 clusters, calculated which cluster is the optimum cluster using Silhouette validation index algorithm. Silhouette Index shows which object well within their cluster and which ones are merely somewhere in between cluster [13]. For each object- $i$ calculate average dissimilarity of object- $i$ with all other within the same cluster $(a(i))$, the smaller value of $a(i)$ the more suitable object- $i$ in the group, then for every cluster repeat average dissimilarity calculation of object- $i$ to all other object in different cluster $(b(i))$ which object- $i$ is not a member. Computation is described in the following formula:

$$
S(i)=\frac{(b(i)-a(i))}{\max \text { 涑 } a(i), b(i)\}}
$$


The closer value $s(i)$ to 1 indicate the less value of $a(i)$, it was concluded object- $i$ have been clustered well, if the value of $s(i)$ is 0 , its means object- $i$ are equally far between two clusters, and if the value of $s(i)$ near to -1 is the worst, since at this position object- $i$ closer to other clusters and better to be a member of its cluster. Number of cluster that have an overall average of $s(i)$ is a cluster with the optimal value or can be formulated as follows:

$$
G S_{u}=\frac{1}{n} \sum_{i=1}^{n} s(i)
$$

(8)

Table 3. Domain values for each of average $R, F, M$

\begin{tabular}{|c|c|c|}
\hline Attribute & $\begin{array}{c}\text { Linguistic } \\
\text { Variable }\end{array}$ & Domain Value \\
\hline \multirow{3}{*}{ Recency } & Long Time Ago & $0 \leq \mathrm{r}<122$ days \\
& A Little Longer & $122<\mathrm{r}<244$ days \\
& Recently & 244 days $<\mathrm{r}$ \\
\hline \multirow{3}{*}{ Frequency } & Seldom & $0 \leq \mathrm{f}<50$ transactions \\
& Rather Frequently & $50<\mathrm{f}<120$ transactions \\
& Often & 120 transactions $<\mathrm{f}$ \\
\hline \multirow{3}{*}{ Monetary } & Low & $0 \leq \mathrm{m}<80$ Mil.Rupiahs \\
& Medium & 80 Mil $<\mathrm{m}<400$ \\
& High & Mil.Rupiahs \\
& \multicolumn{2}{|c}{} \\
\hline
\end{tabular}

Table 4. Description of customer classes

\begin{tabular}{|c|c|c|c|}
\hline \multicolumn{3}{|c|}{ Description of Linguistic Variables } & \multirow{2}{*}{ Class } \\
\hline Recency & Frequency & Monetary & \\
\hline Recently & Seldom & Low & Dormant D \\
\hline Recently & Seldom & Medium & Dormant A \\
\hline Recently & Seldom & High & Occasional A \\
\hline Recently & Rather frequently & Low & Everyday D \\
\hline Recently & Rather frequently & Medium & Golden D \\
\hline Recently & Rather frequently & High & Superstar D \\
\hline Recently & Often & Low & Everyday A \\
\hline Recently & Often & Medium & Golden A \\
\hline Recently & Often & High & Superstar A \\
\hline A Little Longer & Seldom & Low & Dormant E \\
\hline A Little Longer & Seldom & Medium & Dormant B \\
\hline A Little Longer & Seldom & High & Occasional B \\
\hline A Little Longer & Rather frequently & Low & Everyday E \\
\hline A Little Longer & Rather frequently & Medium & Golden E \\
\hline A Little Longer & Rather frequently & High & Superstar E \\
\hline A Little Longer & Often & Low & Everyday B \\
\hline A Little Longer & Often & Medium & Golden B \\
\hline A Little Longer & Often & High & Superstar B \\
\hline Long Time Ago & Seldom & Low & Dormant $\mathrm{F}$ \\
\hline Long Time Ago & Seldom & Medium & Dormant C \\
\hline Long Time Ago & Seldom & High & Occasional C \\
\hline Long Time Ago & Rather frequently & Low & Everyday F \\
\hline Long Time Ago & Rather frequently & Medium & Golden F \\
\hline Long Time Ago & Rather frequently & High & Superstar F \\
\hline Long Time Ago & Often & Low & Everyday C \\
\hline Long Time Ago & Often & Medium & Golden C \\
\hline Long Time Ago & Often & High & Superstar C \\
\hline
\end{tabular}

\subsection{Cluster Analysis}

In this step determine which class have an optimum cluster. Class of customer assess by averaging R, F, M value for each cluster then determine the domain value of $\mathrm{R}, \mathrm{F}, \mathrm{M}$ based on Table 3. Then based on domain value result determine class of customers for each cluster, class of customer is shown in Table 4.

\section{EXPERIMENTS AND RESULTS}

Experiments were done by forming 2 to 6 clusters with changing the value of vigilance parameter $(\rho)$ and noise suppression $(\theta)$.

First experiment is performed using parameters are shown in Table 5. In the first experiment is used low value of vigilance parameter $(\rho)$ and low value of noise $\operatorname{suppression}(\theta)$ and produce two clusters is illustrated by the following scatter plot diagram (for monetary $=\mathrm{x} 10^{3}$ ) in Figure 4 and result for cluster validation is shown in Table 6.

Table 5. Initialization parameter in first experiment

\begin{tabular}{|l|c|r|}
\hline Parameters & Symbol & Initialization Value \\
\hline Fixed Weight & $(a)$ & 10 \\
Fixed Weight & $(b)$ & 10 \\
Fixed Weight & $(c)$ & 0.1 \\
Winning Activation & $(d)$ & 0.9 \\
Small Parameter & $(e)$ & 0 \\
Learning Rate & $(\alpha)$ & 0.6 \\
\hline Vigilance Parameter & $(\rho)$ & 0.9 \\
Noise Suppression & $(\theta)$ & 0.01 \\
\hline Bottom-Up Weight & $\left(b_{i j}\right)$ & 3.0 \\
Top-Down Weight & $\left(t_{j i}\right)$ & 0.0 \\
\hline
\end{tabular}

Table 6. Result overall average Silhouette for the $1^{\text {st }}$ experiment

\begin{tabular}{|l|r|}
\hline Parameters & Result \\
\hline Vigilance Parameter $(\rho)$ & 0.9 \\
Noise Suppression $(\theta)$ & 0.01 \\
Number of clusters formed & 2 Cluster \\
Overall Average Silhouette Value & 0.864 \\
\hline
\end{tabular}

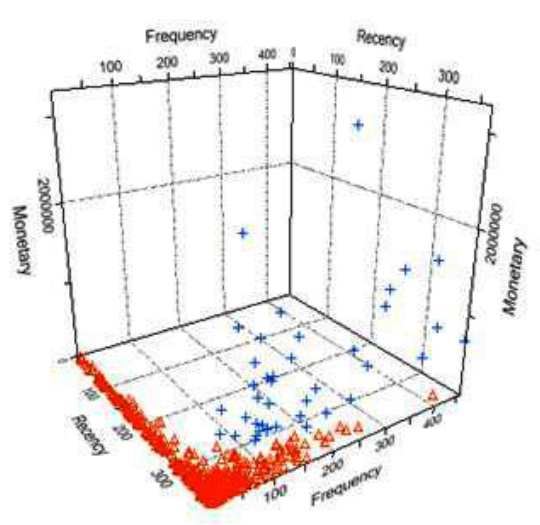

Figure 4. $1^{\text {st }}$ experiment result using scatter plot diagram

In the second experiment, increase the value of vigilance parameter $(\rho)$ becomes 0.985 from previously 0.9 with the same value of noise suppression $(\theta)$ and other parameters (see Table 5), more cluster will be formed as presented in Figure 5 (for monetary $=\times 10^{3}$ ) and result for cluster validation is shown in Table 7 .

If vigilance parameter $(\rho)$ value is increased again becomes 0.989 and with the same value of noise suppression $(\theta)$ and other parameters (see Table 5), still produce 6 cluster but different value of overall average Silhouette as shown in Table 8 and different result of members in each cluster is presented in scatter plot diagram in Figure 6 (for monetary $=$ $\left.\mathrm{x} 10^{3}\right)$. 
Table 7. Result overall average Silhouette for the $2^{\text {nd }}$ experiment

\begin{tabular}{|l|r|}
\hline Parameters & Result \\
\hline Vigilance Parameter $(\rho)$ & 0.985 \\
Noise Suppression $(\theta)$ & 0.01 \\
Number of clusters formed & 6 Cluster \\
Overall Average Silhouette Value & 0.457 \\
\hline
\end{tabular}

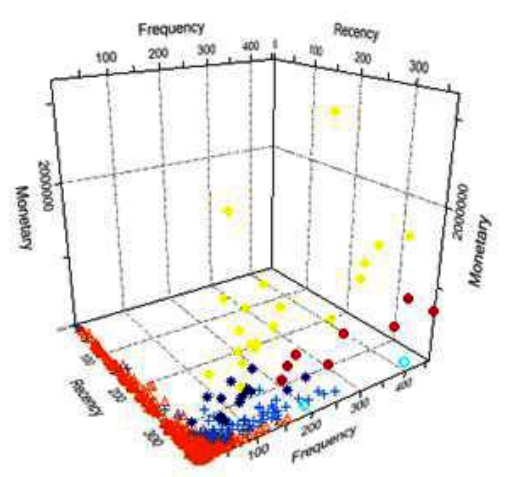

Figure 5. $2^{\text {nd }}$ experiment result using scatter plot diagram

Table 8. Result overall average Silhouette for the $3^{\text {rd }}$ experiment

\begin{tabular}{|l|r|}
\hline Parameters & Result \\
\hline Vigilance Parameter $(\rho)$ & 0.989 \\
Noise Suppression $(\theta)$ & 0.01 \\
Number of clusters formed & 6 Cluster \\
Overall Average Silhouette Value & 0.52 \\
\hline
\end{tabular}

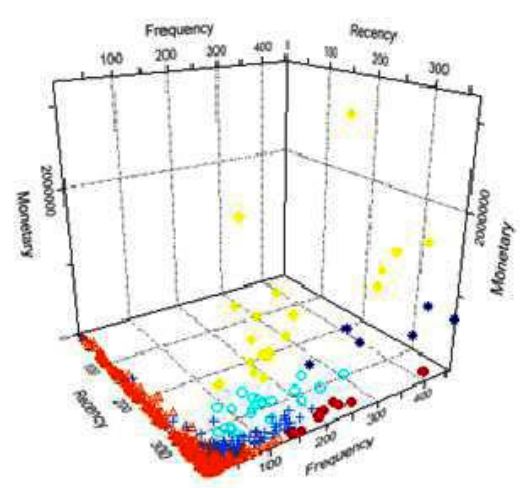

Figure 6. $3^{\text {rd }}$ experiment result using scatter plot diagram

From experiments 2 and 3 were obtained if the value of vigilance parameter $(\rho)$ is improved, the overall Silhouette index value become higher, it means clustering has been done better. Next experiments are performed by increasing the value of noise suppression $(\theta)$ becomes 0.4 with the same vigilance parameter $(\rho)$ to produce 6 cluster formation which is 0.989 and the other parameters that are used remain unchanged (see Table 5). The results are shown in Table 9 and Figure $7\left(\right.$ for monetary $\left.=\times 10^{3}\right)$.

With the same vigilance parameter $(\rho)$ to generate 2 clusters, 0.9 , noise suppression $(\theta) \quad 0.4$ and other parameters remain unchanged (see Table 5) the overall Silhouette result is shown in Table 10 and illustrated of the results in scatter plot diagram shown in Figure 8 (for monetary $=\times 10^{3}$ ).
Table 9. Result overall average Silhouette for the $4^{\text {th }}$ experiment

\begin{tabular}{|l|r|}
\hline Parameters & Result \\
\hline Vigilance Parameter $(\rho)$ & 0.989 \\
Noise Suppression $(\theta)$ & 0.4 \\
Number of clusters formed & 6 Cluster \\
Overall Average Silhouette Value & 0.428 \\
\hline
\end{tabular}

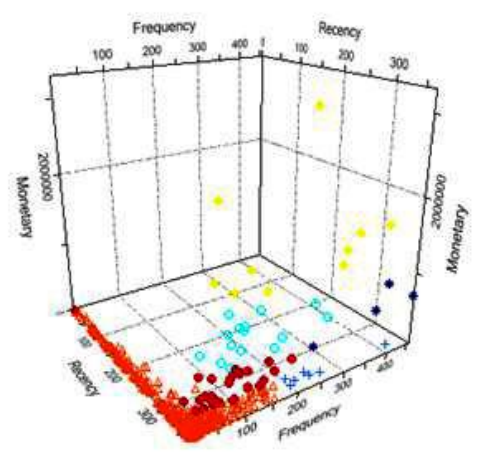

Figure $7.4^{\text {th }}$ experiment result using scatter plot diagram

Table 10. Result overall average Silhouette for the $5^{\text {th }}$ experiment

\begin{tabular}{|l|r|}
\hline Parameters & Result \\
\hline Vigilance Parameter $(\rho)$ & 0.9 \\
Noise Suppression $(\theta)$ & 0.4 \\
Number of clusters formed & 2 Cluster \\
Overall Average Silhouette Value & 0.867 \\
\hline
\end{tabular}

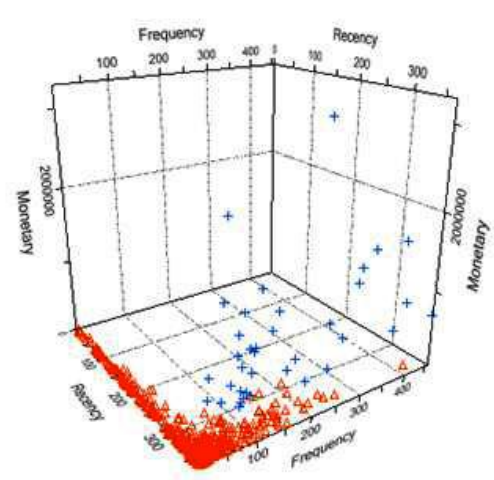

Figure 8. $5^{\text {th }}$ experiment result using scatter plot diagram

Table 11. Result summarized

\begin{tabular}{|c|c|c|c|}
\hline $\begin{array}{c}\text { Number } \\
\text { of } \\
\text { Cluster }\end{array}$ & $\begin{array}{c}\text { Noise } \\
\text { Suppression } \\
(\theta)\end{array}$ & $\begin{array}{c}\text { Vigilance } \\
\text { Parameter }(\rho)\end{array}$ & $\begin{array}{c}\text { Overall Average } \\
\text { Silhouette Value }\end{array}$ \\
\hline 6 & 0.01 & 0.985 & 0.457 \\
\hline 6 & 0.01 & 0.989 & 0.52 \\
\hline 6 & 0.4 & 0.989 & 0.428 \\
\hline 5 & 0.01 & 0.981 & 0.428 \\
\hline 5 & 0.4 & 0.975 & 0.399 \\
\hline 4 & 0.01 & 0.9715 & 0.676 \\
\hline 4 & 0.4 & 0.956 & 0.752 \\
\hline 3 & 0.01 & 0.96875 & 0.704 \\
\hline 3 & 0.4 & 0.94 & 0.728 \\
\hline 2 & 0.01 & 0.9 & 0.864 \\
\hline 2 & 0.4 & 0.9 & 0.867 \\
\hline
\end{tabular}


Table 12. Result cluster analysis

\begin{tabular}{|l|l|l|}
\hline \multirow{2}{*}{} & \multicolumn{2}{|c|}{ Result } \\
\cline { 2 - 3 } & $1^{\text {st }}$ Cluster & $2^{\text {nd }}$ Cluster \\
\hline Average R & 302.1 & 360.5 \\
Linguistic Variable R & Recently & Recently \\
\hline Average F & 25.6 & 153.0 \\
Linguistic Variable F & Seldom & Often \\
\hline Average M & 57311704.2 & 1022288536.3 \\
Linguistic Variable M & Low & High \\
\hline Final Class Customer & Dormant D & Superstar A \\
\hline
\end{tabular}

The result of overall average Silhouette index for vigilance parameter $(\rho)$ 0.989, noise suppression $(\theta) \quad 0.01$ which produces six clusters is higher than with the equal vigilance parameter $(\rho)$, noise suppression $(\theta) 0.4$ which also produced 6 clusters, but for vigilance parameter $(\rho) \quad 0.9$, noise suppression $(\theta) 0.01$ which produce two clusters have lower overall average Silhouette index value than equal vigilance parameter $(\rho)$ with noise suppression $(\theta) 0.4$ which also produces two clusters, so the good selection of parameters can either increase the overall average value of Silhouette index. Results to produce 2 to 6 clusters are summarized in Table 11 .

Judging from the experiment results, were obtained two to six clusters, the overall average Silhouette index was highest when produce two clusters with noise suppression $(\theta) 0.4$ and vigilance parameter $(\rho) 0.9$ which is 0.867 , so for two clusters formed analysis to know customer classes is shown in Table 12. From the analysis based on linguistic variable values (Table 3 and Table 4) was obtained, first cluster is Dormant D class which is the third lowest class with low level of Frequency and Monetary even though recently did transaction, the second cluster is Superstar A, which is the highest class with high Recency, Frequency and Monetary.

\section{CONCLUSION AND FUTURE WORK}

This paper has successfully demonstrated the use of RFM model combine with ART 2 algorithm. In experiments to produce 2 to 6 clusters were obtained, 2 clusters formed have the largest value of overall average Silhouette index which is 0.867. All clusters formed have overall average Silhouette index value more than 0 especially for 2, 3, 4 clusters which have overall average Silhouette index value close to 1 , indicates most of the Silhouette value in all clusters were formed have a positive value or above zero, its means ART2 clustering algorithm which produces 2 to 6 clusters have been able clustering well.

For future development, experiments can be improved with algorithm to generate the rule [5] and use more than one cluster validation index algorithm to obtain better optimum cluster [6] and expected application of data mining tools for customer segmentation can be done on a various kind of data.

\section{ACKNOWLEDGMENTS}

Our thank goes to Department of Information Technology Udayana University, Bali, Indonesia who has helped organize this research.

\section{REFERENCES}

[1] Tsiptsis, Kontantinos. Chorianopoulos, Antonios. 2009 Data Mining Techniques in CRM: Inside Customer Segmentation. United Kingdom: John Wiley \& Sons, Ltd

[2] Zumstein, D., 2007, "Customer Performance Measurement: Analysis of the Benefit of a Fuzzy Classification Approach in Customer Relationship Management" (Thesis), Switzerland: University of Fribourg

[3] Shashidhar, H.V., Subramanian V., 2011, Customer Segmentation of Bank based on Data Mining - Security Value based Heuristic Approach as a Replacement to Kmeans Segmentation, International Journal of Computer Applications, Vol. 19, No. 8

[4] Shankar S., Dr.Purusothaman T., Kannimuthu, S. Vishnu, P.K., 2010, A Novel Utility and Frequency Based Itemset Mining Approach for Improving CRM in Retail Business. International Journal of Computer Applications, Vol. 1, No. 16

[5] Cheng, C.H, Chen, Y.S, 2009, classifying the segmentation of customer value via RFM model and RS theory, Expert Systems with Applications, 36, 41764184

[6] Yohana, N., 2011, Data mining dengan metode fuzzy untuk Customer Relationship Management (CRM) pada perusahaan retail, Master Thesis, Graduate Program, Udayana University, Bali, Indonesia.

[7] Gemala, Y., 2011, Segmentasi pelanggan dengan algoritma K-Means dan analisa RFM untuk mendukung strategi pengelolaan pelanggan di PT. Indosat Mega Media, Undergraduate Thesis, Sepuluh November Institute of Technology

[8] Chen, C.H., Khoo, L.P., Yan, W., 2002, A strategy for acquiring customer requirement patterns using laddering technique and ART2 neural network, Advanced Engineering Informatics, 16, 229-240

[9] Hughes, A.M., 1994, Strategic database marketing, Chicago: Probus Publishing Company.

[10] Carpenter, G., Grossberg, S., 1987, ART2: stable selforganization of pattern recognition codes for analog input patterns. Appl Optics 26: 4919-30.

[11] Fausset, L., 1993. Fundamental of Neural Network: Architecture, Algorithms, and Application, Precentice Hall

[12] Carpenter, G., Grossberg, S., The art of adaptive recognition by a self-organizing neural network, Computer 1988, 21, 77-88

[13] Rousseeuw, P.J., "Silhouettes: a graphical aid to the interpretation and validation of cluster analysis", J. Comp App. Math, Vol. 20, 1987, pp. 53-65 